\title{
REVSED The genome sequence of a stonefly, Nemurella pictetii
}

\section{Klapalek, 1900 [version 2; peer review: 2 approved, 1 approved}

\section{with reservations]}

\author{
Craig R. Macadam¹, Caleala Clifford², Benjamin W. Price (i)3, \\ Natural History Museum Genome Acquisition Lab, \\ Darwin Tree of Life Barcoding collective, \\ Wellcome Sanger Institute Tree of Life programme, \\ Wellcome Sanger Institute Scientific Operations: DNA Pipelines collective, \\ Tree of Life Core Informatics collective, Darwin Tree of Life Consortium \\ ${ }^{1}$ Buglife - The Invertebrate Conservation Trust, Stirling, UK \\ ${ }^{2}$ South East Environmental Assessment and Advice Team, Natural Resources Wales, Cardiff, UK \\ ${ }^{3}$ Department of Life Sciences, Natural History Museum, London, UK
}

V2 First published: 15 Feb 2022, 7:56

https://doi.org/10.12688/wellcomeopenres.17684.1

Latest published: 25 Feb 2022, 7:56

https://doi.org/10.12688/wellcomeopenres.17684.2

\section{Abstract}

We present a genome assembly from an individual male Nemurella pictetii (Arthropoda; Insecta; Plecoptera; Nemouridae). The genome sequence is 257 megabases in span. The majority of the assembly $(99.79 \%)$ is scaffolded into 12 chromosomal pseudomolecules, with the $X$ sex chromosome assembled. The $X$ chromosome was found at half coverage, but no Y chromosome was found. The mitochondrial genome was assembled, and is $16.0 \mathrm{~kb}$ in length.

\section{Keywords}

Nemurella pictetii, genome sequence, chromosomal, Plecoptera

This article is included in the Tree of Life gateway.

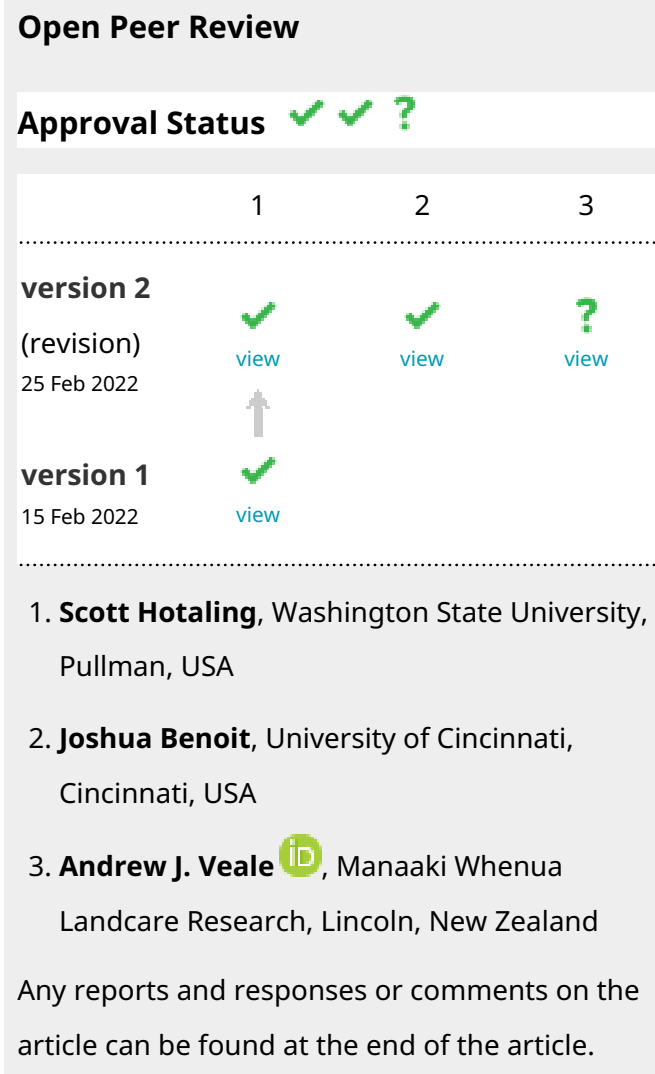

1. Scott Hotaling, Washington State University, Pullman, USA

2. Joshua Benoit, University of Cincinnati, Cincinnati, USA

3. Andrew J. Veale ${ }^{D}$, Manaaki Whenua Landcare Research, Lincoln, New Zealand Any reports and responses or comments on the article can be found at the end of the article. 
Corresponding author: Darwin Tree of Life Consortium (mark.blaxter@sanger.ac.uk)

Author roles: Macadam CR: Investigation, Resources, Writing - Original Draft Preparation; Clifford C: Investigation, Resources; Price BW: Writing - Review \& Editing;

Competing interests: No competing interests were disclosed.

Grant information: This work was supported by the Wellcome Trust through core funding to the Wellcome Sanger Institute (206194) and the Darwin Tree of Life Discretionary Award (218328).

The funders had no role in study design, data collection and analysis, decision to publish, or preparation of the manuscript.

Copyright: @ 2022 Macadam CR et al. This is an open access article distributed under the terms of the Creative Commons Attribution License, which permits unrestricted use, distribution, and reproduction in any medium, provided the original work is properly cited.

How to cite this article: Macadam CR, Clifford C, Price BW et al. The genome sequence of a stonefly, Nemurella pictetii Klapalek, 1900 [version 2; peer review: 2 approved, 1 approved with reservations] Wellcome Open Research 2022, 7:56

https://doi.org/10.12688/wellcomeopenres.17684.2

First published: 15 Feb 2022, 7:56 https://doi.org/10.12688/wellcomeopenres.17684.1 


\section{REVISED Amendments from Version 1}

We thank the reviewer for his comments on the manuscript, and have made minor changes in response. We have also corrected some additional errors in Table 1, which listed incorrect BioSample and BioProject accession numbers.

Any further responses from the reviewers can be found at the end of the article

\section{Species taxonomy}

Eukaryota; Metazoa; Ecdysozoa; Arthropoda; Hexapoda; Insecta; Pterygota; Neoptera; Polyneoptera; Plecoptera; Nemouroidea; Nemouridae; Nemourinae; Nemurella; Nemurella pictetii Klapalek, 1900 (NCBI:txid143722).

\section{Background}

Nemurella pictetii is a western palearctic species found from northern Spain to Siberia, and throughout the British Isles, although appears to be less common in Scotland. It is considered a eurytherm and can be found in a variety of habitats from rivers and streams to ponds and lakes. It does not appear to have any altitudinal preference; however, in Ireland it is more usually encountered in streams draining peaty soils usually at higher altitudes and in small headwater streams and seepages (Baars \& Kelly-Quinn, 2006; Hynes, 1977). In Great Britain and Europe, larvae are known to occur in small upland lakes with stony shorelines and in vegetated ponds, wetlands and marshes (e.g. Lillehammer (1975), Macadam (2015), Wolf \& Zwick (1989)). Larvae are often common and can be numerous in small trickles and streams with dense vegetation, woody material and/or organic matter (Costello et al., 1984; Feeley et al., 2019; Graf et al., 2009; Wolf \& Zwick, 1989), but generally they are scarce, only occurring in low densities. They are also highly tolerant of low pH and conductivity (Baars \& Kelly-Quinn, 2006; Feeley, 2012; Murphy et al., 2013). Both Brittain (1991) and Baars \& Kelly-Quinn (2006) report this species as somewhat tolerant of nutrient enrichment. Larvae are opportunistic feeders utilising a broad range of food sources, but preferring biofilm (Lieske \& Zwick, 2007).

Nemurella pictetii is the sole representative of the genus Nemurella. The high-quality genome sequence described here is, to our knowledge, the first one reported for N. pictetii. This assembly, generated as part of the Darwin Tree of Life project, also represents a high-quality addition to an underrepresented taxon (Plecoptera) and wider group of insects (aquatic insects) (Hotaling et al., 2020). The genome sequence for $N$. pictetii will aid in understanding the biology, physiology and ecology of the species.

\section{Genome sequence report}

The genome was sequenced from one male $N$. pictetii (Figure 1) collected from River Taff Fawr, Garwnant, Wales (latitude 51.8082, longitude -3.4449). A total of 68-fold coverage in Pacific Biosciences single-molecule long reads and 183-fold coverage in 10X Genomics read clouds were generated. Primary assembly contigs were scaffolded with chromosome conformation Hi-C data. Manual assembly curation corrected 62 missing/misjoins and removed 8 haplotypic duplications, reducing the assembly size by $2.35 \%$ and scaffold number by $65.52 \%$ and increasing the scaffold N50 by $8.35 \%$.

The final assembly has a total length of $257 \mathrm{Mb}$ in 20 sequence scaffolds with a scaffold N50 of $24.6 \mathrm{Mb}$ (Table 1). The majority of the assembly sequence (99.79\%) was assigned to 12 chromosomal-level scaffolds, representing 11 autosomes (numbered by sequence length), and the $\mathrm{X}$ sex chromosome (Figure 1-Figure 4; Table 2). The sex of the specimen was

Table 1. Genome data for Nemurella pictetii, ipNemPict2.1.

\begin{tabular}{|l|l|}
\hline Project accession data & \\
\hline Assembly identifier & ipNemPict2.1 \\
\hline Species & Nemurella pictetii \\
\hline Specimen & $\begin{array}{l}\text { ipNemPict2 (male, genome } \\
\text { assembly); ipNemPict1 } \\
\text { (unknown sex, Hi-C) }\end{array}$ \\
\hline NCBI taxonomy ID & NCBI:txid143722 \\
\hline BioProject & PRJEB47468 \\
\hline BioSample ID & SAMEA7520996 \\
\hline Isolate information & $\begin{array}{l}\text { Male, whole organism } \\
\text { (ipNemPict2); unknown sex, } \\
\text { whole organism (ipNemPict1) }\end{array}$ \\
\hline Raw data accessions & \\
\hline PacificBiosciences SEQUEL II & ERR6808073, ERR6909090, \\
\hline ERR6939286, ERR6939287
\end{tabular}

*BUSCO scores based on the insecta_odb10 BUSCO set using v5.1.2. $\mathrm{C}=$ complete $[\mathrm{S}=$ single copy, $\mathrm{D}=$ duplicated], $\mathrm{F}=$ fragmented, $\mathrm{M}=$ missing, $\mathrm{n}=$ number of orthologues in comparison. A full set of BUSCO scores is available at https://blobtoolkit.genomehubs.org/view/ipNemPict2.1/ dataset/CAKLCZ01/busco. 


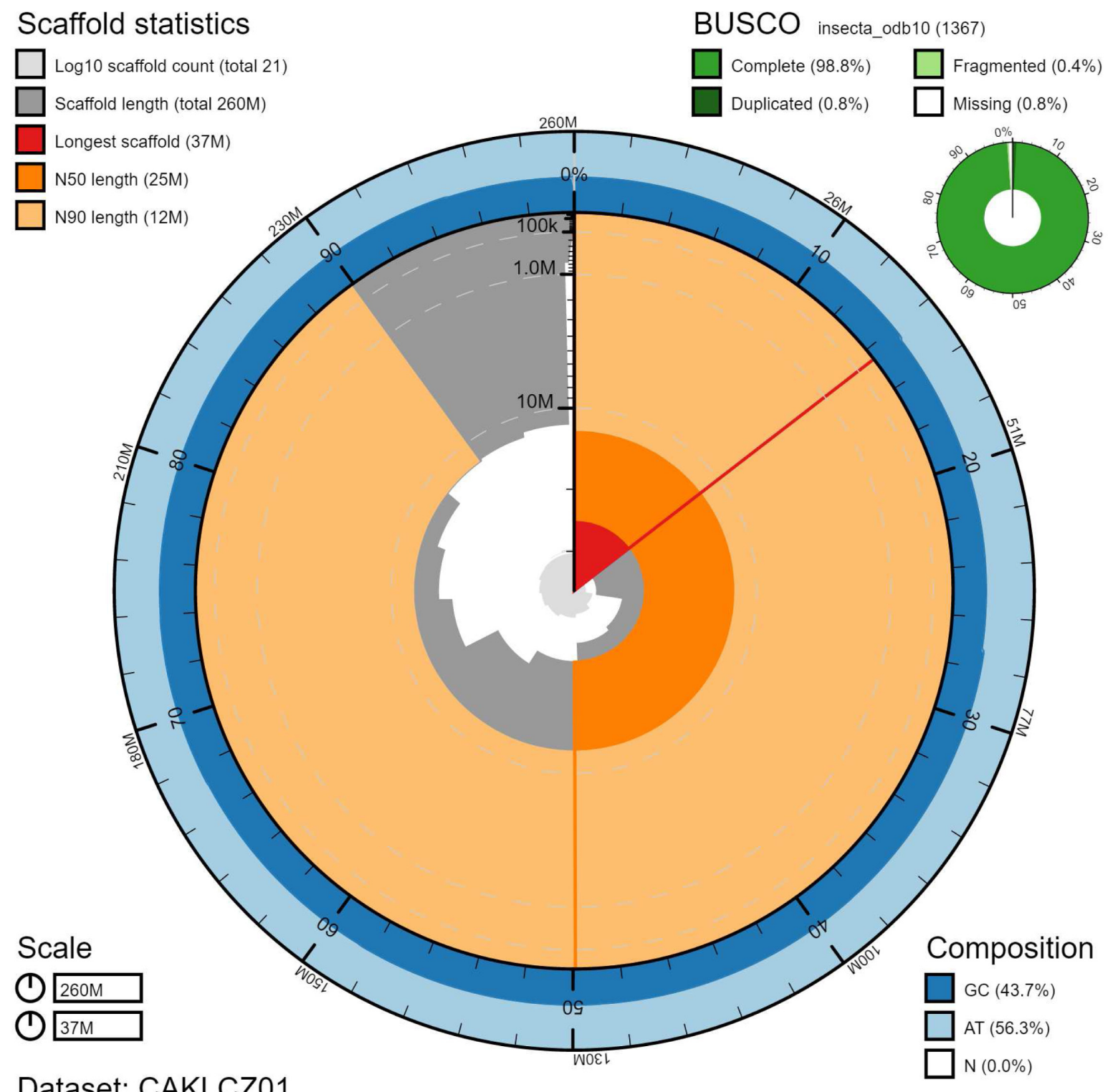

\section{Dataset: CAKLCZ01}

Figure 1. Genome assembly of Nemurella pictetii, ipNemPict2.1: metrics. The BlobToolKit Snailplot shows N50 metrics and BUSCO gene completeness. The main plot is divided into 1,000 size-ordered bins around the circumference with each bin representing $0.1 \%$ of the $257,052,056$ bp assembly. The distribution of scaffold lengths is shown in dark grey with the plot radius scaled to the longest scaffold present in the assembly (37,341,160 bp, shown in red). Orange and pale-orange arcs show the N50 and N90 scaffold lengths $(24,564,665$ and $12,448,030$ bp), respectively. The pale grey spiral shows the cumulative scaffold count on a log scale with white scale lines showing successive orders of magnitude. The blue and pale-blue area around the outside of the plot shows the distribution of GC, AT and $\mathrm{N}$ percentages in the same bins as the inner plot. A summary of complete, fragmented, duplicated and missing BUSCO genes in the insecta_odb10 set is shown in the top right. An interactive version of this figure is available at https://blobtoolkit.genomehubs.org/view/ CAKLCZ01/dataset/CAKLCZ01/snail.

determined to be male as chromosome $\mathrm{X}$ was found at half coverage, despite no evidence of the presence of a $\mathrm{Y}$ chromosome. The assembly has a BUSCO v5.1.2 (Manni et al., 2021) completeness of $98.8 \%$ (single $98.0 \%$, duplicated $0.8 \%$ ) using the insecta_odb10 reference set $(n=1,367)$. While not fully phased, the assembly deposited is of one haplotype. Contigs corresponding to the second haplotype have also been deposited.

\section{Methods}

Sample acquisition and DNA extraction

Two $N$. pictetii specimens (ipNemPict1, unknown sex, and ipNemPict2, male) were collected from River Taff Fawr, Garwnant, Wales (latitude 51.8082, longitude -3.4449) by Natural Resources Wales using a kick-net. The sample was identified by representatives of the same body and snap-frozen in liquid nitrogen. Unfortunately, no images of the samples are available.

Page 4 of 13 


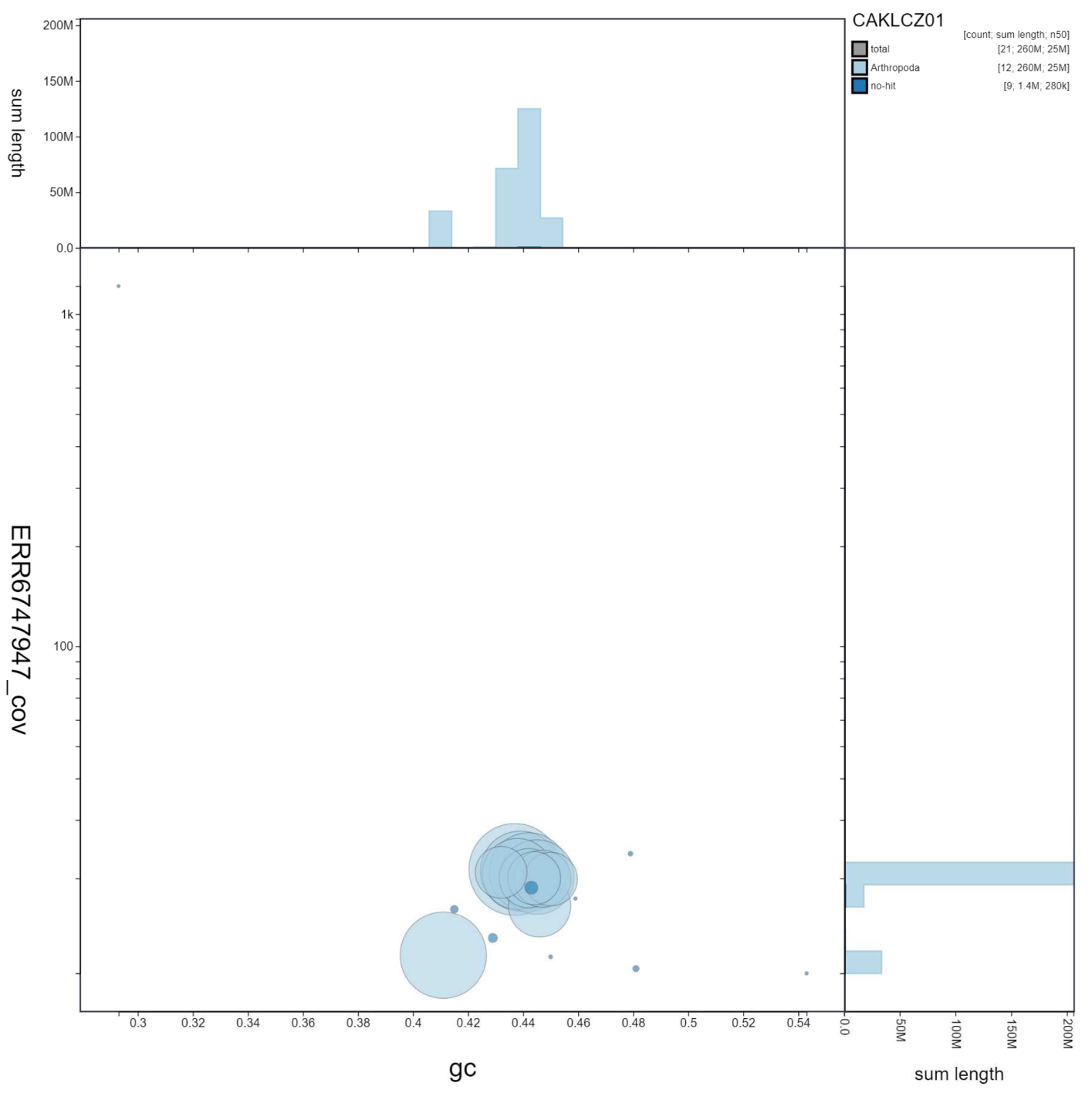

Figure 2. Genome assembly of Nemurella pictetii, ipNemPict2.1: GC coverage. BlobToolKit GC-coverage plot. Scaffolds are coloured by phylum. Circles are sized in proportion to scaffold length Histograms show the distribution of scaffold length sum along each axis. An interactive version of this figure is available at https://blobtoolkit.genomehubs.org/view/CAKLCZ01/dataset/CAKLCZ01/blob.

DNA was extracted at the Tree of Life laboratory, Wellcome Sanger Institute. The ipNemPict2 sample was weighed and dissected on dry ice. Whole organism tissue was cryogenically disrupted to a fine powder using a Covaris cryoPREP Automated Dry Pulveriser, receiving multiple impacts. Fragment size analysis of $0.01-0.5 \mathrm{ng}$ of DNA was then performed using an Agilent FemtoPulse. High molecular weight (HMW) DNA was extracted using the Qiagen MagAttract HMW DNA extraction kit. Low molecular weight DNA was removed from a 200-ng aliquot of extracted DNA using 0.8X AMpure XP purification kit prior to $10 \mathrm{X}$ Chromium sequencing; a minimum of $50 \mathrm{ng}$ DNA was submitted for $10 \mathrm{X}$ sequencing. HMW DNA was sheared into an average fragment size between $12-20 \mathrm{~kb}$ in a Megaruptor 3 system with speed setting 30. Sheared DNA was purified by solid-phase reversible immobilisation using AMPure PB beads with a $1.8 \mathrm{X}$ ratio of beads to sample to remove the shorter fragments and concentrate the DNA sample. The concentration of the sheared and purified DNA was assessed using a Nanodrop spectrophotometer and Qubit Fluorometer and Qubit dsDNA High Sensitivity Assay kit. Fragment size distribution was evaluated by running the sample on the FemtoPulse system.

\section{Sequencing}

Pacific Biosciences HiFi circular consensus and 10X Genomics Chromium read cloud sequencing libraries were constructed according to the manufacturers' instructions. Sequencing was performed by the Scientific Operations core at the Wellcome Sanger Institute on Pacific Biosciences SEQUEL II (HiFi) and Illumina NovaSeq 6000 (10X) instruments. Hi-C data were generated from whole organism tissue of ipNemPict1 using the Arima Hi-C+ kit and sequenced on an Illumina HiSeq $\mathrm{X}$ instrument.

\section{Genome assembly}

Assembly was carried out with Hifiasm (Cheng et al., 2021); haplotypic duplication was identified and removed with 


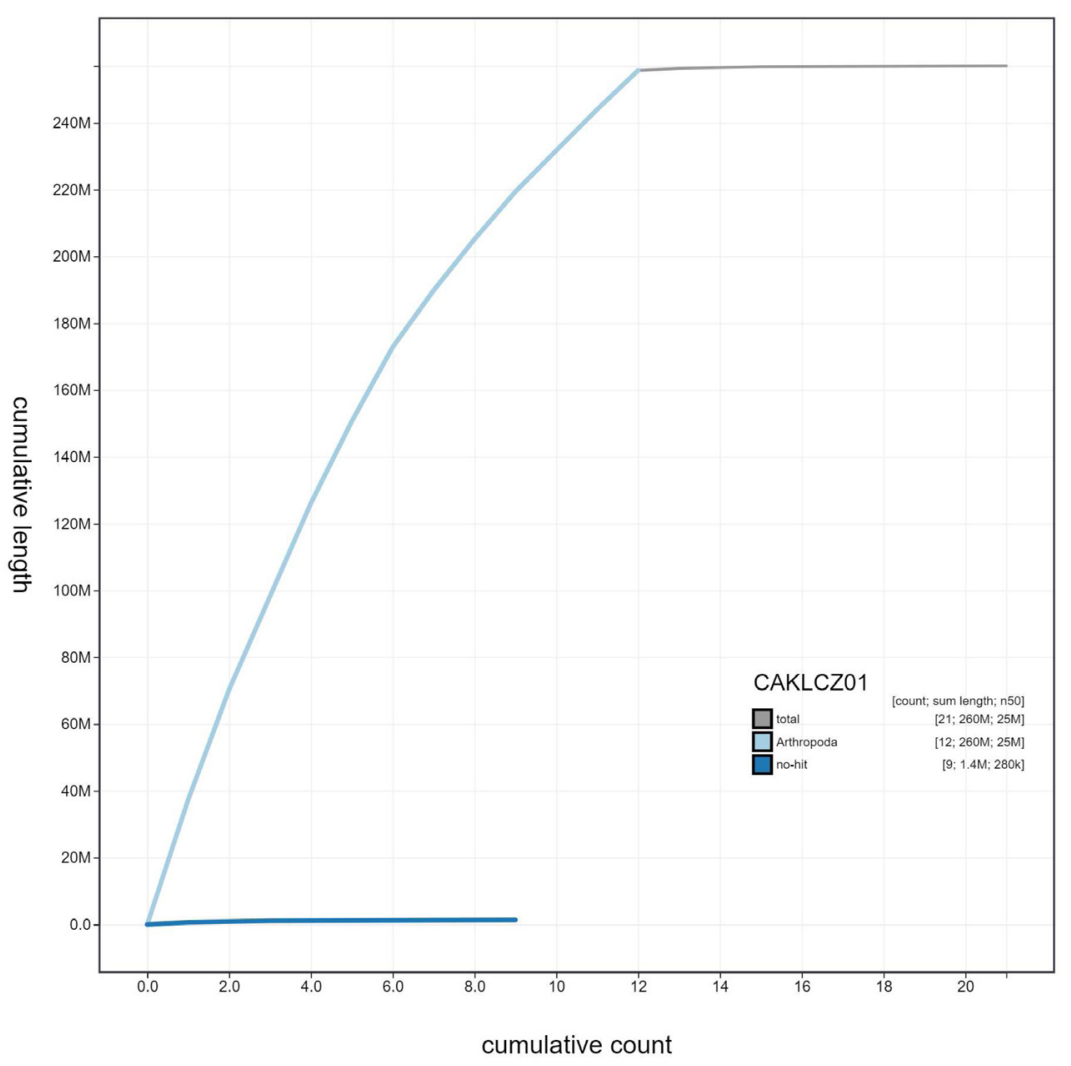

Figure 3. Genome assembly of Nemurella pictetii, ipNemPict2.1: cumulative sequence. BlobToolKit cumulative sequence plot. The grey line shows cumulative length for all scaffolds. Coloured lines show cumulative lengths of scaffolds assigned to each phylum using the buscogenes taxrule. An interactive version of this figure is available at https://blobtoolkit.genomehubs.org/view/CAKLCZ01/dataset/ CAKLCZ01/cumulative.

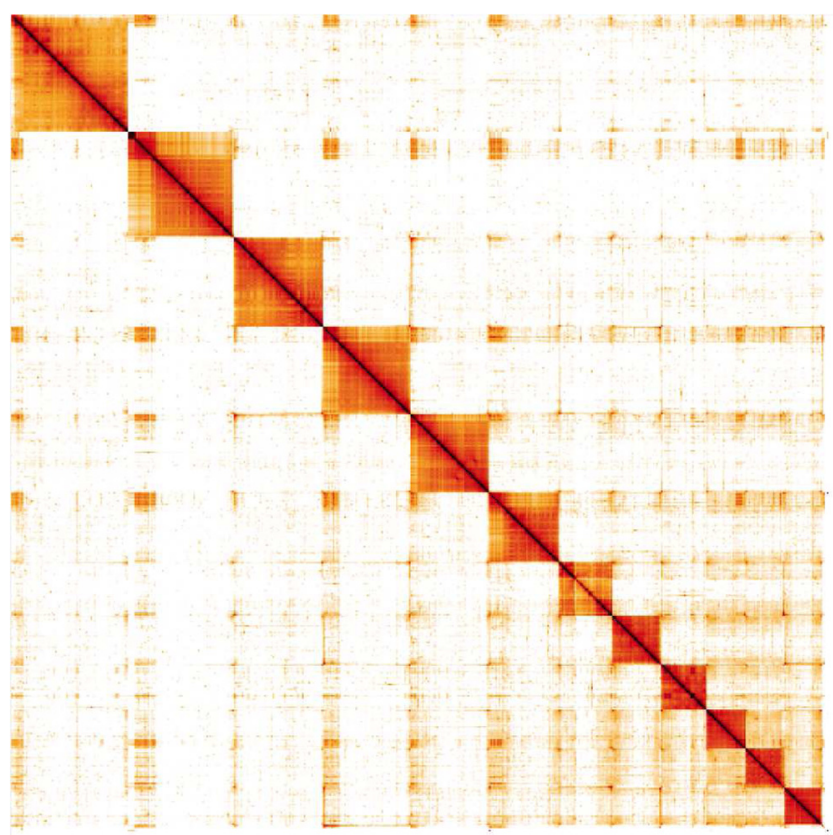

Figure 4. Genome assembly of Nemurella pictetii, ipNemPict2.1: Hi-C contact map. Hi-C contact map of the ipNemPict2.1 assembly, visualised in HiGlass. Chromosomes are shown in order of size from left to right and top to bottom. 
purge_dups (Guan et al., 2020). One round of polishing was performed by aligning $10 \mathrm{X}$ Genomics read data to the assembly with longranger align, calling variants with freebayes (Garrison \& Marth, 2012). The assembly was then scaffolded with Hi-C data (Rao et al., 2014) using SALSA2 (Ghurye et al., 2019). The assembly was checked for contamination as described previously (Howe et al., 2021). Manual curation (Howe et al., 2021) was performed using HiGlass (Kerpedjiev et al., 2018) and Pretext. The mitochondrial genome was assembled using MitoHiFi (Uliano-Silva et al., 2021), which performs annotation using MitoFinder (Allio et al., 2020). The genome was analysed and BUSCO scores generated within the BlobToolKit environment (Challis et al., 2020). Table 3 contains a list of all software tool versions used, where appropriate.

\section{Ethics/compliance issues}

The materials that have contributed to this genome note have been supplied by a Darwin Tree of Life Partner. The submission of materials by a Darwin Tree of Life Partner is subject to the Darwin Tree of Life Project Sampling Code of Practice. By agreeing with and signing up to the Sampling Code of Practice, the Darwin Tree of Life Partner agrees they will meet the legal and ethical requirements and standards set out within this document in respect of all samples acquired for, and

\begin{tabular}{|c|c|c|c|}
\hline INSDC accession & Chromosome & Size (Mb) & GC\% \\
\hline OV121115.1 & 1 & 37.34 & 43.7 \\
\hline OV121117.1 & 2 & 28.23 & 43.9 \\
\hline OV121118.1 & 3 & 27.56 & 44.2 \\
\hline OV121119.1 & 4 & 24.56 & 44.5 \\
\hline OV121120.1 & 5 & 22.19 & 43.8 \\
\hline OV121121.1 & 6 & 17.00 & 44.6 \\
\hline OV121122.1 & 7 & 15.30 & 44.2 \\
\hline OV121123.1 & 8 & 14.27 & 44.7 \\
\hline OV121124.1 & 9 & 12.45 & 45.0 \\
\hline OV121125.1 & 10 & 12.20 & 44.4 \\
\hline OV121126.1 & 11 & 11.63 & 43.2 \\
\hline OV121116.1 & $x$ & 32.95 & 41.1 \\
\hline OV121127.1 & MT & 0.02 & 29.3 \\
\hline- & Unplaced & 1.35 & 44.3 \\
\hline
\end{tabular}

Table 3. Software tools used.

\begin{tabular}{|l|l|l|}
\hline Software tool & Version & Source \\
\hline Hifiasm & 0.15 .3 & Cheng et al., 2021 \\
\hline purge_dups & 1.2 .3 & Guan et al., 2020 \\
\hline SALSA2 & 2.2 & Ghurye et al., 2019 \\
\hline Iongranger align & 2.2 .2 & $\begin{array}{l}\text { https://support.10xgenomics.com/genome-exome/ } \\
\text { software/pipelines/latest/advanced/other-pipelines }\end{array}$ \\
\hline freebayes & $1.3 .1-17$-gaa2ace8 & Garrison \& Marth, 2012 \\
\hline MitoHiFi & 2.0 & Uliano-Silva et al., 2021 \\
\hline HiGlass & 1.11 .6 & Kerpedjiev et al., 2018 \\
\hline PretextView & $0.2 . x$ & https://github.com/wtsi-hpag/PretextView \\
\hline BlobToolKit & 2.6 .4 & Challis et al., 2020 \\
\hline
\end{tabular}


supplied to, the Darwin Tree of Life Project. Each transfer of samples is further undertaken according to a Research Collaboration Agreement or Material Transfer Agreement entered into by the Darwin Tree of Life Partner, Genome Research Limited (operating as the Wellcome Sanger Institute), and in some circumstances other Darwin Tree of Life collaborators.

\section{Data availability}

European Nucleotide Archive: Nemoura pictetii. Accession number PRJEB47468; https://identifiers.org/ena.embl/ PRJEB47468 [identifiers.org]

The genome sequence is released openly for reuse. The $N$. pictetii genome sequencing initiative is part of the Darwin Tree of Life (DToL) project. All raw sequence data and the assembly have been deposited in INSDC databases. The genome will be annotated and presented through the Ensembl pipeline at the European Bioinformatics Institute. Raw data and assembly accession identifiers are reported in Table 1.
Author information

Members of the Natural History Museum Genome Acquisition Lab are listed here: https://doi.org/10.5281/zenodo.5746819.

Members of the Darwin Tree of Life Barcoding collective are listed here: https://doi.org/10.5281/zenodo.5744972.

Members of the Wellcome Sanger Institute Tree of Life programme are listed here: https://doi.org/10.5281/zenodo.5744840.

Members of Wellcome Sanger Institute Scientific Operations: DNA Pipelines collective are listed here: https://doi.org/10.5281/ zenodo.5746904.

Members of the Tree of Life Core Informatics collective are listed here: https://doi.org/10.5281/zenodo.5743293.

Members of the Darwin Tree of Life Consortium are listed here: https://doi.org/10.5281/zenodo.5638618.
Allio R, Schomaker-Bastos A, Romiguier J, et al.: MitoFinder: Efficient Automated Large-Scale Extraction of Mitogenomic Data in Target Enrichment Phylogenomics. Mol Ecol Resour. 2020; 20(4): 892-905. PubMed Abstract | Publisher Full Text | Free Full Text

Baars JR, Kelly-Quinn M: The Plecoptera of Irish Freshwaters--Species Distribution, Status and Association with Environmental Parameters. Report to The Heritage Council of Ireland, Wildlife Grant Scheme, 2006; 1-26. Brittain JE: Life History Characteristics as a Determinant of the Response of Mayflies and Stoneflies to Man-Made Environmental Disturbance (Ephemeroptera and Plecoptera). Overview and Strategies of Ephemeroptera and Plecoptera. Sandhill Crane Press. Gainesville, Fla. USA, 1991.

Reference Source

Challis R, Richards E, Rajan J, et al.: BlobToolKit - Interactive Quality Assessment of Genome Assemblies. G3 (Bethesda). 2020; 10(4): 1361-74. PubMed Abstract | Publisher Full Text | Free Full Text

Cheng $\mathrm{H}$, Concepcion GT, Feng X, et al.: Haplotype-Resolved de Novo Assembly Using Phased Assembly Graphs with Hifiasm. Nat Methods. 2021; 18(2): 170-75.

PubMed Abstract | Publisher Full Text | Free Full Text

Costello MJ, McCarthy TK, O'Farrell MM: The Stoneflies (Plecoptera) of the Corrib Catchment Area, Ireland. Annls Limnol. 1984; 20(1-2): 25-34. Publisher Full Text

Feeley HB, Little R, Baars JR: Some Additional Notes on the Life Histories of Nemurella Pictetii Klapálek and Brachyptera Risi (Morton) (Plecoptera) in Ireland. Ir Nat J. 2019; 36(2): 146-147.

Reference Source

Feeley HB: The Impact of Mature Conifer Forest Plantations on the Hydrochemical and Ecological Quality of Headwater Streams in Ireland, with Particular Reference to Episodic Acidification. University College Dublin. 2012.

Garrison E, Marth G: Haplotype-Based Variant Detection from Short-Read Sequencing. arXiv: 1207.3907, 2012

Reference Source

Ghurye J, Rhie A, Walenz BP, et al.: Integrating Hi-C Links with Assembly Graphs for Chromosome-Scale Assembly. PLoS Comput Biol. 2019; 15(8): e1007273.

PubMed Abstract | Publisher Full Text | Free Full Text

Graf W, Lorenz AW, de Figueroa JMT, et al.: Plecoptera. Coronet Books Incorporated. 2009.

Reference Source

Guan D, McCarthy SA, Wood J, et al.: Identifying and Removing Haplotypic Duplication in Primary Genome Assemblies. Bioinformatics. 2020; 36(9): 2896-2898.

PubMed Abstract | Publisher Full Text | Free Full Text
Hotaling S, Kelley JL, Frandsen PB: Aquatic Insects Are Dramatically Underrepresented in Genomic Research. Insects. 2020; 11(9): 601. PubMed Abstract | Publisher Full Text | Free Full Text

Howe K, Chow W, Collins J, et al.: Significantly Improving the Quality of Genome Assemblies through Curation. GigaScience. 2021; 10(1): giaa153. PubMed Abstract | Publisher Full Text | Free Full Text

Hynes HBN: A Key to the Adults and Nymphs of the British Stoneflies (Plecoptera): With Notes on Their Ecology and Distribution. Freshwater Biological Association, 1977; 17.

Reference Source

Kerpedjiev P, Abdennur N, Lekschas F, et al.: HiGlass: Web-Based Visual Exploration and Analysis of Genome Interaction Maps. Genome Biol. 2018; 19(1): 125.

PubMed Abstract | Publisher Full Text | Free Full Text

Lieske R, Zwick P: Food Preference, Growth and Maturation of Nemurella Pictetii (Plecoptera: Nemouridae). Freshw Biol. 2007; 52(7): 1187-97. Publisher Full Text

Lillehammer A: Norwegian Stoneflies. III. Field Studies on Ecological Factors Influencing Distribution. NorJ Entomol. 1975; 22: 71-80.

Macadam CR: A Review of the Stoneflies (Plecoptera) of Great Britain: Species Status No.20. Commissioned Report NECR174. Natural England. 2015.

Reference Source

Manni M, Berkeley MR, Seppey M, et al:: BUSCO Update: Novel and Streamlined Workflows along with Broader and Deeper Phylogenetic Coverage for Scoring of Eukaryotic, Prokaryotic, and Viral Genomes. $\mathrm{Mol}$ Biol Evol. 2021; 38(10): 4647-54.

PubMed Abstract | Publisher Full Text | Free Full Text

Murphy JF, Davy-Bowker J, McFarland B, et al.: A Diagnostic Biotic Index for Assessing Acidity in Sensitive Streams in Britain. Ecol Indic. 2013; 24: 562-72.

Publisher Full Text

Rao SS, Huntley MH, Durand NC, et al:: A 3D Map of the Human Genome at Kilobase Resolution Reveals Principles of Chromatin Looping. Cell. 2014; 159(7): 1665-80.

PubMed Abstract | Publisher Full Text | Free Full Text

Uliano-Silva M, Nunes JGF, Krasheninnikova K, et al.: marcelauliano/MitoHiFi: mitohifi_v2.0. 2021

Publisher Full Text

Wolf B, Zwick P: Plurimodal Emergence and Plurivoltinism of Central European Populations of Nemurella Pictetii (Plecoptera: Nemouridae). Oecologia. 1989; 79(4): 431-38.

PubMed Abstract | Publisher Full Text 


\section{Open Peer Review}

\section{Current Peer Review Status:}

\section{Version 2}

Reviewer Report 12 May 2022

https://doi.org/10.21956/wellcomeopenres.19633.r49820

(C) 2022 Veale A. This is an open access peer review report distributed under the terms of the Creative Commons Attribution License, which permits unrestricted use, distribution, and reproduction in any medium, provided the original work is properly cited.

\section{Andrew J. Veale}

Manaaki Whenua Landcare Research, Lincoln, New Zealand

In the Background section, "although appears to be less common in Scotland" should be "although $\underline{i t}$ appears to be less common in Scotland".

In the methods you should identify what life stage each of the individuals was - are these adults or juveniles, and if juvenile, which instar?

In stonefly genomics I have found issues with contamination from filamentous algae and diatoms in particular, which you appear not to have had issues with. When you say whole organism tissue what do you mean? Was the gut present? Was the outer cuticle present? My assumption is that you had an adult and dissected out the gut but it would be good to confirm.

Were any tissues stored for museum accession?

Was species ID confirmed through mitochondrial sequences?

Having an XO sex determination system appears relatively common in Plecoptera, as it appears to be in this species ${ }^{1}$. This should probably be made explicit.

I have no issues with the genome assembly and methods therein - it looks like a high quality assembly.

Good luck!

\section{References}

1. Blackmon H, Ross L, Bachtrog D: Sex Determination, Sex Chromosomes, and Karyotype Evolution in Insects.J Hered. 108 (1): 78-93 PubMed Abstract | Publisher Full Text

\section{Is the rationale for creating the dataset(s) clearly described?}


Yes

Are the protocols appropriate and is the work technically sound?

Yes

Are sufficient details of methods and materials provided to allow replication by others? Partly

Are the datasets clearly presented in a useable and accessible format?

Yes

Competing Interests: No competing interests were disclosed.

Reviewer Expertise: Genomics - have previously worked on Plecoptera genomics.

I confirm that I have read this submission and believe that I have an appropriate level of expertise to confirm that it is of an acceptable scientific standard, however I have significant reservations, as outlined above.

Reviewer Report 09 May 2022

https://doi.org/10.21956/wellcomeopenres.19633.r50257

(C) 2022 Benoit J. This is an open access peer review report distributed under the terms of the Creative Commons Attribution License, which permits unrestricted use, distribution, and reproduction in any medium, provided the original work is properly cited.

\section{Joshua Benoit}

Department of Biological Sciences, University of Cincinnati, Cincinnati, OH, USA

This study provides the details on the genome sequence of a stonefly, Nemurella pictetii. No additional comments as issues found by the other reviewer have been addressed. Sufficient background and information is provided for this genome. The genome is high quality and will provide a new resource for an understudied system.

Is the rationale for creating the dataset(s) clearly described?

Yes

Are the protocols appropriate and is the work technically sound?

Yes

Are sufficient details of methods and materials provided to allow replication by others? Yes

Are the datasets clearly presented in a useable and accessible format?

Yes 
Competing Interests: No competing interests were disclosed.

Reviewer Expertise: Arthropod biology

I confirm that I have read this submission and believe that I have an appropriate level of expertise to confirm that it is of an acceptable scientific standard.

Reviewer Report 02 March 2022

https://doi.org/10.21956/wellcomeopenres.19633.r48912

(C) 2022 Hotaling S. This is an open access peer review report distributed under the terms of the Creative Commons Attribution License, which permits unrestricted use, distribution, and reproduction in any medium, provided the original work is properly cited.

\section{Scott Hotaling}

School of Biological Sciences, Washington State University, Pullman, WA, USA

Approved.

Is the rationale for creating the dataset(s) clearly described?

Yes

Are the protocols appropriate and is the work technically sound? Yes

Are sufficient details of methods and materials provided to allow replication by others? Yes

Are the datasets clearly presented in a useable and accessible format? Yes

Competing Interests: No competing interests were disclosed.

Reviewer Expertise: Insect genomics, genome biology, aquatic ecology

I confirm that I have read this submission and believe that I have an appropriate level of expertise to confirm that it is of an acceptable scientific standard.

\section{Version 1}

Reviewer Report 18 February 2022

https://doi.org/10.21956/wellcomeopenres.19566.r48724 
(c) 2022 Hotaling S. This is an open access peer review report distributed under the terms of the Creative Commons Attribution License, which permits unrestricted use, distribution, and reproduction in any medium, provided the original work is properly cited.

\section{Scott Hotaling}

1 School of Biological Sciences, Washington State University, Pullman, WA, USA

${ }^{2}$ School of Biological Sciences, Washington State University, Pullman, WA, USA

In this study, Macadam and colleagues report the assembly of a high-quality stonefly genome the best to date for this entire order of insects. The study and methods contain no notable flaws and I see no issues in terms of its scientific robustness.

\section{Background:}

One missing component - placing this genome assembly in context for the group and insects as a whole. It fills a major niche for Plecoptera and adds a key species for aquatic insects broadly which are dramatically underrepresented (see my article Hotaling et al. 2020 $\left.{ }^{1}\right)$. These points can be highlighted for the reader. It might also be useful to note that the three previous stonefly genomes are exceptionally poor-quality and represent perhaps the lowest quality group of assemblies for any insect order (see my article Hotaling et al. 2021, Figure $1 d^{2}$ ). Adding these references is far from critical to the report's effectiveness. They would simply be "value added" for a future reader seeking broader context in terms of insect genomics.

Also there are double parentheses in the first paragraph of the section in the PDF, mid-way through. These may need correction.

\section{Genome sequence report:}

Described as being a female specimen in the first sentence but later as male. This should be confirmed/corrected.

\section{Genome assembly:}

There is a space missing in the PDF between "Table 3" and "contains" on the top of pg. 7.

In this study, Macadam and colleagues report the assembly of a high-quality stonefly genome-the best to date for this entire order of insects.

\section{References}

1. Hotaling S, Kelley JL, Frandsen PB: Aquatic Insects Are Dramatically Underrepresented in Genomic Research.Insects. 2020; 11 (9). PubMed Abstract | Publisher Full Text

2. Hotaling S, Sproul J, Heckenhauer J, Powell A, et al.: Long Reads Are Revolutionizing 20 Years of Insect Genome Sequencing. Genome Biology and Evolution. 2021; 13 (8). Publisher Full Text

\section{Is the rationale for creating the dataset(s) clearly described?}

Yes

Are the protocols appropriate and is the work technically sound?

Yes 
Are sufficient details of methods and materials provided to allow replication by others? Yes

Are the datasets clearly presented in a useable and accessible format?

Yes

Competing Interests: No competing interests were disclosed.

Reviewer Expertise: Insect genomics, genome biology, aquatic ecology

I confirm that I have read this submission and believe that I have an appropriate level of expertise to confirm that it is of an acceptable scientific standard. 